\title{
Dynamics of Polymer Sheets Cutting Mechanism
}

\author{
Livija Cveticanin* - Ratko Maretic - Miodrag Zukovic \\ Faculty of Technical Sciences, Novi Sad, Serbia
}

In the paper the dynamics of a cutting mechanism for polymer sheets is analyzed. The mechanism contains two connected slider-crank mechanisms which transform the rotating motion of the leading element into a straightforward motion of the output slider. The mechanism is driven by an electro motor and the slider represents the cutting tool. The cutting force is required to be constant. Using this assumption the kinematic and dynamic properties of the mechanism are determined. In particular, the influence of the cutting force on the input angular velocity of the leading element is analyzed. In addition, the interaction of geometrical and dynamical properties of the mechanism and of the cutting force is investigated. Angular velocity is a function of the cutting force, damping and inertia properties of the system. Variation of the angular velocity of the driving motor are calculated analytically and numerically. Analytically obtained results are in a good agreement with numerical ones.

Keywords: two joined slider-crank mechanism, kinematic and dynamic analysis, cutting force, non-ideal forcing

\section{O INTRODUCTION}

A great variety of mechanisms, tools and devices are made for cutting through materials based on specific requirements connected with the properties of the cutting object, its dimensions and form or strength and elasticity, as well as on the characteristics of the cutting tool and the driving motor [1]. Most of these tools are analysed, discussed and shown in textbooks for mechanical engineers and technicians. They all have a simple construction in common. For example, for cutting of the parts of strings, rods or bands, which represent the continual cutting object, the cutting mechanism may be based on the four-bar one (see [2]).

In this paper a mechanism for throughout cutting of the polymer sheet, which represents the discontinual cutting object, is considered. Due to elastic properties of the polymer sheet and its tendency to crumple, and also to sheet dimensions, it was required that cutting be done with a one-direction cutting force. This was possible by a translatory motion of the cutting tool. As the driving was with an electro motor, the mechanism had to transform the rotating motion of the leading element into a translatory motion of the leaded element. The mechanism which transforms the rotation into straight motion is the slider-crank mechanism. This mechanism and its modifications have been widely analyzed and applied to internal combustion engines and other various purposes (see for example [3] to [6]). In this paper, due to its simplicity the slider-crank mechanism is assumed as a basic one for the cutting device. Joining together two slider-crank mechanisms an appropriate device is obtained which also transforms the rotating motion of the leading element into translatory motion of the slider which is connected with a cutting tool. The idea of joining of two slider-crank mechanisms is not a new one. The double-slider crank mechanisms are already used in air compressors [7], two piston pumps [8], in the cutting machine for elliptical cylinder [9], in the two-side piston engine [10], in the haptic devices to generate pulling or pushing motion [11] and [12], in robotics [13] to [16], and also as a continuous casting mold oscillation device [17].

In Section 1 the structural synthesis of the cutting mechanism is considered. The advantages and disadvantages of the cutting mechanism based on the two slider-crank mechanism in comparison to the slider-crank mechanisms (simple and eccentric) are discussed. In Section 2 kinematic properties of the cutting mechanism are analyzed. In Section 3 the mathematical description of the mechanism's motion is given and in Section 4 a dynamic analysis is done. The obtained results are discussed in Section 5.

\section{STRUCTURAL SYNTHESIS OF THE CUTTING MECHANISM}

The structure of the cutting mechanism is required to satisfy the following:

- the mechanism has to transform the input rotating motion into a translatory one,

- the cutting element has to move translatorily,

- the cutting process has to be during motion of the cutting element from up to down.

To fulfill these requirements, in this paper a device which contains two slider-crank mechanisms is suggested (see Fig.1). The system is designed to have an eccentric $\mathrm{O}_{1} \mathrm{AB}$ and a simple $\mathrm{O}_{2} \mathrm{DE}$ slidercrank mechanism both of which are connected with a rod $\mathrm{BC}$. The leading element of the mechanism is the crankshaft $\mathrm{O}_{1} \mathrm{~A}$, while the slider is the cutting tool at point $\mathrm{E}$. The suggested mechanism converts the 
rotating motion of the crankshaft $\mathrm{O}_{1} \mathrm{~A}$ into a straightline motion of the slider E.

The mechanism has the following elements: $\mathrm{O}_{1} \mathrm{~A}$ $=a, \mathrm{AB}=b, \mathrm{BC}=c, \mathrm{O}_{2} \mathrm{C}=r, \mathrm{O}_{2} \mathrm{D}=g, \mathrm{DE}=h$.

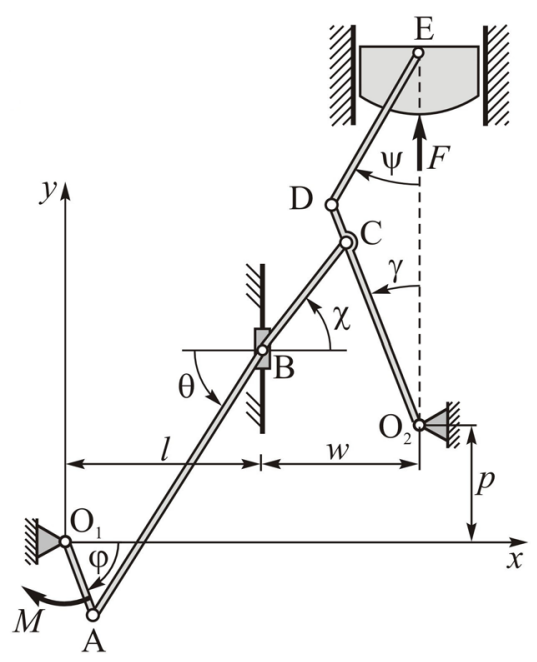

Fig. 1. Model of the cutting mechanism

From Fig. 1 the position of the slider $\mathrm{B}$ of the eccentric slider-crank mechanism $\mathrm{O}_{1} \mathrm{AB}$ (see Fig. 1) is given with the coordinates:

$$
\begin{gathered}
x_{B}=a \cos \varphi+b \cos \theta \equiv l, \\
y_{B}=-a \sin \varphi+b \sin \theta .
\end{gathered}
$$

Eliminating $\theta$ in Eqs. (1) and (2) the position of the slider $\mathrm{B}$ as a function of the leading angle $\varphi$ is obtained:

$$
y_{B}=-a \sin \varphi+b \sqrt{1-\left(\frac{l-a \cos \varphi}{b}\right)^{2}} .
$$

For the simple slider-crank mechanism $\mathrm{O}_{2} \mathrm{DE}$ (see Fig. 1) the translatory motion of the slider is described as:

$$
y_{E}=g \cos \gamma+h \cos \psi,
$$

where the relation between the angles $\gamma$ and $\psi$ is given with the expression:

$$
g \sin \gamma=h \sin \psi .
$$

Substituting Eq. (5) into Eq. (4) the following is obtained:

$$
y_{E}=g \cos \gamma+h \sqrt{\left(1-\frac{g^{2}}{h^{2}}\right)+\frac{g^{2}}{h^{2}} \cos ^{2} \gamma},
$$

which describes the position of the slider $\mathrm{E}$ as a function of the leading angle $\gamma$ of the slider-crank mechanism $\mathrm{O}_{2} \mathrm{DE}$.
Let us make a connection between these two slider-crank mechanisms. Due to the fact that after the connection with the rod BC the two slider-crank mechanism remains an one-degree-of-freedom system (as it was the case for the simple and eccentric slidercrank mechanisms), the relation between the position of the slider $\mathrm{E}$ and leading angle $\varphi$ of the crankshaft $\mathrm{O}_{1}$ A needs to be determined.

From Fig. 1 it is evident that the position of the slider $\mathrm{E}$ in the coordinate system $x \mathrm{O}_{1} y$ is:

$$
y=p+y_{E} .
$$

Moreover,

$$
\begin{gathered}
w=c \cos \chi+r \sin \gamma . \\
y_{B}+c \sin \chi=p+r \cos \gamma .
\end{gathered}
$$

Eliminating $\chi$ in Eqs. (8) and (9) the $y_{B}-\gamma$ i.e., $\varphi-\gamma$ expression is obtained as:

$$
\begin{gathered}
\left(c^{2}-w^{2}-r^{2}-\left(p-y_{B}\right)^{2}-2 r\left(p-y_{B}\right) \cos \gamma\right)^{2}= \\
=4 w^{2} r^{2}\left(1-\cos ^{2} \gamma\right),
\end{gathered}
$$

i.e.,

$$
A_{2} \cos ^{2} \gamma-A_{1} \cos \gamma+A_{0}=0,
$$

where

$$
\begin{aligned}
& A=c^{2}-w^{2}-r^{2}-\left(p-y_{B}\right)^{2}, A_{0}=A^{2}-4 w^{2} r^{2}, \\
& A_{1}=4 A r\left(p-y_{B}\right), A_{2}=4 r^{2}\left(\left(p-y_{B}\right)^{2}+w^{2}\right),
\end{aligned}
$$

and $p$ is a constant distance between fixed points $\mathrm{O}_{1}$ and $\mathrm{O}_{2}$ in $y$ direction. Solving the quadratic equation (11) for $\cos \gamma$ and substituting into Eqs. (7) with (6), the $y-\varphi$ relation follows.

\subsection{Comparison of the Simple, Eccentric and Two Slider- Crank Mechanisms}

In Fig. 2 the displacement-angle relations for: a) simple (Eq. (6)), b) eccentric (Eq. (3)) and c) two slider-crank (Eq. (7)) mechanisms are plotted. It is assumed that for the simple and eccentric slidercrank mechanism the length of the leading shaft $(0.8$ $\mathrm{m})$ and of the connecting rod $(0.32 \mathrm{~m})$ are equal for both mechanisms and the eccentricity is $0.20 \mathrm{~m}$. The dimensions of the two joined slider-crank mechanisms in $\mathrm{m}$ are: $a=0.8, b=0.32, c=0.14, r=0.20, g=$ $0.24, h=0.18, l=0.20, p=0.12, w=0.16$ and the cutting depth is $\delta=0.12$. In our consideration the common assumption used for comparing the three mechanisms is that the cutting depth has to be equal and the cutting angle is calculated from the lowest position of the slider. In Fig. 2 the full line indicates the motion of the slider in the sheet (where the shaded 
area is for cutting) and the dotted line shows the motion of the slider out of the sheet. Comparing the diagrams in Fig. 2, it can be concluded:

- Cutting takes longer with the simple and eccentric slider-crank mechanism than with the two joined slider-crank mechanism.

- The interval in which the slider (cutting tool) is above the cutting object is much longer for the two joined slider-crank mechanism than for the simple and eccentric one. During this period the manipulation with the cutting sheet may be completed. This, however, is not the case for the simple and eccentric slider-crank mechanisms. Namely, the 'resting' period for the simple and eccentric slider-crank mechanisms is extremely short and does not give the opportunity to finish the manipulation with the sheet: setting and its removing from the machine.

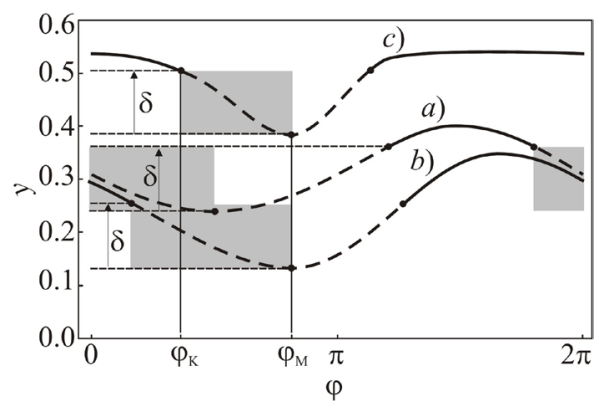

Fig. 2. $y$ - $\varphi$ diagrams for a) simple slider-crank mechanism, b) eccentric slider-crank mechanism, c) two-joined slider-crank mechanism (shaded area-cutting, dotted line-slider in the sheet, full line-slider out of sheet)

It is the reason that the joined two-slider-crank mechanism is introduced and assumed for the cutting process. During one period of motion of the twojoined slider-crank mechanism the manipulation with the polymer sheet and also the cutting proces is possible to be finished.

\section{KINEMATICS OF THE CUTTING MECHANISM}

Let us determine the velocity $v_{E}$ of the cutting tool as a function of the angular velocity $\dot{\varphi}$ of the leading crankshaft. Using the relations Eqs. (6) and (7) the velocity of the cutting tool is:

$$
v_{E} \equiv \dot{y}=-\dot{\gamma} \frac{g y_{E} \sin \gamma}{y_{E}-g \cos \gamma} .
$$

The time derivative of (10) gives $\dot{\gamma}\left(\dot{y}_{B}\right)$ as:

$$
\begin{gathered}
\dot{\gamma} r\left(w \cos \gamma-\left(p-y_{B}\right) \sin \gamma\right)= \\
=\left(p-y_{B}+r \cos \gamma\right) \dot{y}_{B},
\end{gathered}
$$

where according to Eq. (3)

$$
\dot{y}_{B}=-a \dot{\varphi} \frac{y_{B} \cos \varphi+l \sin \varphi}{y_{B}+a \sin \varphi} .
$$

Substituting Eq. (14) with Eq. (15) into Eq. (13) the velocity of the slider as the function of the angular velocity of the leading crankshaft is obtained:

$$
\begin{gathered}
v_{E}=a \dot{\varphi} f(\varphi), \\
f(\varphi)=\frac{g}{r} \cdot \frac{y_{E} \sin \gamma}{y_{E}-g \cos \gamma} . \\
\cdot \frac{p-y_{B}+r \cos \gamma}{w \cos \gamma-\left(p-y_{B}\right) \sin \gamma} \cdot \frac{y_{B} \cos \varphi+l \sin \varphi}{y_{B}+a \sin \varphi} .
\end{gathered}
$$

Function $f(\varphi)$ is periodical with a period of $2 \pi$.

\section{MATHEMATICAL MODEL OF THE MECHANISM}

The considered two slider-crank mechanism has one degree of freedom and the generalized coordinate is the angle $\varphi$ of the leading crank $\mathrm{O}_{1} \mathrm{~A}$. The Lagrange differential equation of motion of the mechanism for the generalized coordinate $\varphi$ is in general:

$$
\frac{d}{d t} \frac{\partial T}{\partial \dot{\varphi}}-\frac{\partial T}{\partial \varphi}+\frac{\partial \Phi}{\partial \dot{\varphi}}=Q_{\varphi},
$$

where $T$ is the kinetic energy of the mechanism, $\Phi$ is the dissipative function and $Q_{\varphi}$ is the generalized force.

It is assumed that the mass of the cutting tool is $m$ and the moment of inertia of the leading element is $J$. The inertial properties of other elements in mechanism can be omitted in comparison to the previous. Then, the kinetic energy of the mechanism is a sum of the kinetic energy of the cutting tool and of the leading element:

$$
T=\frac{1}{2} J \dot{\varphi}^{2}+\frac{1}{2} m v_{E}^{2},
$$

where $v_{E}$ is the velocity of cutting tool given with Eq. (16). Substituting Eq. (16) into Eq. (19) we obtain:

$$
T=\frac{1}{2} J \dot{\varphi}^{2}+\frac{1}{2} m a^{2} f^{2} \dot{\varphi}^{2},
$$

where the kinetic energy is the function of the angular velocity $\dot{\varphi}$. 
Since all the mechanism members are rigid, the elastic energy of the system is zero.

The mechanism is driven by an electro motor whose characteristics is that the driving torque $M$ is the function of the velocity $\dot{\varphi},[18]$ :

$$
M=M_{0}\left(1-\frac{\dot{\varphi}}{\omega_{0}}\right),
$$

where $M_{0}=$ const. and $\omega_{0}$ is the synchrone angular velocity of the motor. Thereby, the driving load is expressed as a function of the angular coordinate describing the crank rotation. Physically this means that the motion of the mechanism has an influence on the motor torque. Such a mechanism is subjected to non-ideal forcing (see [19 to 21]).

The cutting process is required to be managed during the motion of the cutting tool from up to down in the angle interval $\left[\varphi_{K}, \varphi_{M}\right]$ where $\varphi_{M}$ corresponds to the lowest position of the cutting tool which satisfies the relation $d y\left(\varphi_{M}\right) / \mathrm{d} \varphi=0$ and $\varphi_{K}$ is the angle position for which the cutting starts and has to be adopted to the thickness of the sheet $\delta: y\left(\varphi_{K}\right)=y\left(\varphi_{M}\right)+\delta$. In this interval the cutting force is required to be constant and sufficiently strong to provide the cutting without folding of the sheet. Otherwise, the cutting force is assumed to be zero. Mathematically, for $\varphi \in\left[\varphi_{K}, \varphi_{M}\right]$ the constant force is $F=F_{0}$ and for $\varphi \in\left[0, \varphi_{K}\right) \cup\left(\varphi_{M}, 2 \pi\right]$ it is $F=0$.

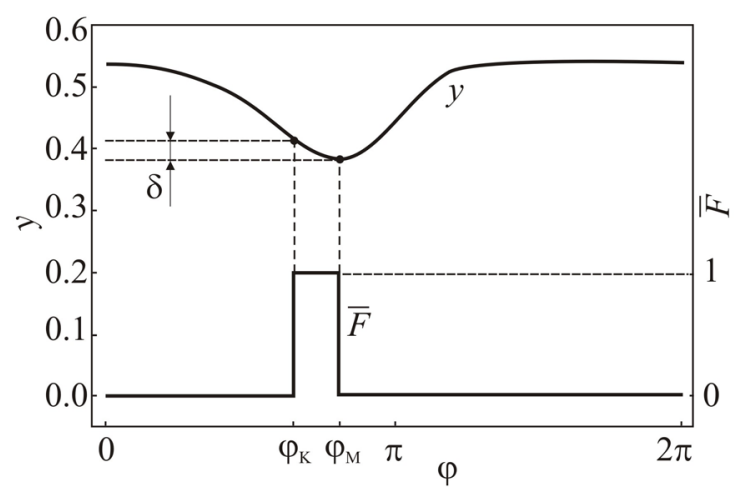

Fig. 3. $y-\varphi$ and $\bar{F}-\varphi$ diagrams of the cutting tool

As the cutting process is periodical, the cutting force is modeled as a UnitStep function:

$$
\begin{gathered}
F \equiv F(\varphi)=F_{0} \bar{F}(\varphi)= \\
=F_{0}\left(\operatorname{UnitStep}\left(\bmod (\varphi, 2 \pi)-\varphi_{K}\right)-\right. \\
\left.- \text { UnitStep }\left(\bmod (\varphi, 2 \pi)-\varphi_{M}\right)\right),
\end{gathered}
$$

where the unit step function is defined as:

$$
\operatorname{UnitStep}(x)=\left\{\begin{array}{ll}
1, & x \geq 0 \\
0, & x<0
\end{array} .\right.
$$

The force distribution is plotted in Fig. $3\left(\varphi_{K}=\right.$ 2.06379, $\varphi_{M}=2.55591, \delta=0.03$ ).

The driving torque $M$ and the cutting force $F$ give the virtual works for a virtual angle and displacement variations, respectively, i.e.,

$$
\delta A=M \delta \varphi+F \delta y .
$$

According to Eq. (16) the variation of the variable $y$ is:

$$
\delta y=a f \delta \varphi .
$$

Substituting Eq. (24) into Eq. (23) we obtain $\delta A=Q_{\varphi} \delta \varphi$ where the generalized force is:

$$
Q_{\varphi}=M+a f F .
$$

During cutting the damping force acts. For energy dissipation during the slider motion through various materials of the polymer sheet, the damping force is assumed to be proportional to the velocity of the cutting tool, i.e.,

$$
\vec{F}_{w}=-q \vec{v}_{E} .
$$

The corresponding dissipative function is:

$$
\Phi=\frac{1}{2} q v_{E}^{2},
$$

where $q$ is the damping coefficient. According to Eq. (16), the dissipative function Eq. (24) is:

$$
\Phi=\frac{1}{2} q a^{2} f^{2} \dot{\varphi}^{2} .
$$

Substituting Eqs. (15), (20) and (28) and the corresponding derivatives calculated in Appendix into Eq. (18), the differential equation of motion is obtained:

$$
\begin{gathered}
\left(J+m a^{2} f^{2}\right) \ddot{\varphi}+m a^{2} f \frac{d f}{d \phi} \dot{\varphi}^{2}+q a^{2} f^{2} \dot{\varphi}= \\
=M(\dot{\varphi})+a f F(\varphi),
\end{gathered}
$$

where $f$ and $(\mathrm{d} f / \mathrm{d} \varphi)$ are $\varphi$ - periodical functions with period of $2 \pi$. (see Eqs. (17) and (A.5)).

According to Eqs. (17) and (A.5), the functions $f(\varphi), d f(\varphi) / d \varphi$ and $f(\varphi)(d f(\varphi) / d \varphi)$ are plotted in Fig. 4.

Introducing the dimensionless values:

$$
\begin{gathered}
\tau=\omega_{0} t, \quad I=\frac{J \omega_{0}^{2}}{M_{0}}, \quad I=\frac{J \omega_{0}^{2}}{M_{0}}, \\
\lambda=\frac{F_{0} a}{M_{0}}, \quad Q=\frac{q a^{2} \omega_{0}}{M_{0}}, \quad \mu=\frac{m a^{2} \omega_{0}^{2}}{M_{0}},
\end{gathered}
$$


the differential equation (29) transforms into:

$$
\begin{gathered}
\left(I+\mu f^{2}\right) \varphi^{\prime \prime}+\mu f \frac{d f}{d \varphi}\left(\varphi^{\prime}\right)^{2}+Q f^{2} \varphi^{\prime}= \\
=\left(1-\varphi^{\prime}\right)+\lambda f \bar{F}(\varphi), \\
\varphi^{\prime}=d \varphi / d \tau
\end{gathered}
$$

where $\varphi^{\prime}=d \varphi / d \tau$ and $\varphi^{\prime \prime}=d^{2} \varphi / d \tau^{2}, \mu$ is dimensionless mass of the cutting tool, $I$ is dimensionless moment of inertia of the leading crank $\mathrm{O}_{1} \mathrm{~A}, Q$ is the dimensionless damping coefficient, $\lambda$ is dimensionless cutting force and $\tau$ is the dimensionless time. Differential equation (31) is strong nonlinear one and only for some special parameter values the closed form analytical solution is possible to be obtained. Otherwise, the Eq. (31) is solved numerically using the Runge-Kutta procedure.

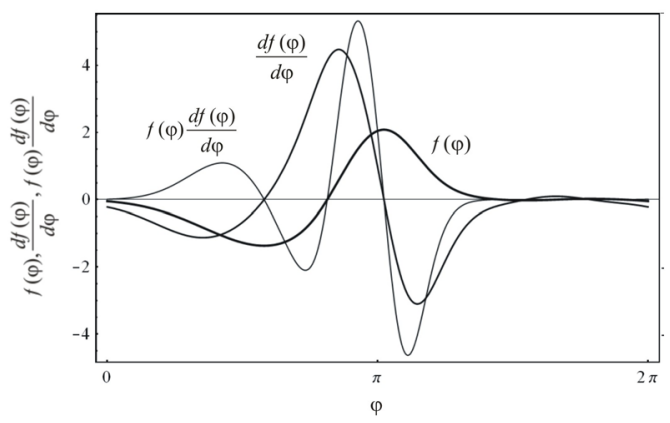

Fig. 4. $f(\varphi)-\varphi$, $(d f / d \varphi)-\varphi$ and $f(\varphi)(d f(\varphi) / d \varphi)-\varphi$ curves

\section{DYNAMIC ANALYSES}

Solving Eq. (31) for various values of $\bar{F}(\varphi)$ the influence of the cutting force on the angular velocity of the motor is obtained. In Fig. 5 the $\varphi^{\prime}-\tau$ curves for various values of $\bar{F}(\varphi)$ are plotted. (Dimensionless parameters are $\lambda=0.033, I=1.4557 \cdot 10^{-4}$, $\mu=1.051 \cdot 10^{-3}, Q=0.00134$ and the initial conditions $\varphi(0)=0$ and $\left.\varphi^{\prime}(0)=1\right)$.

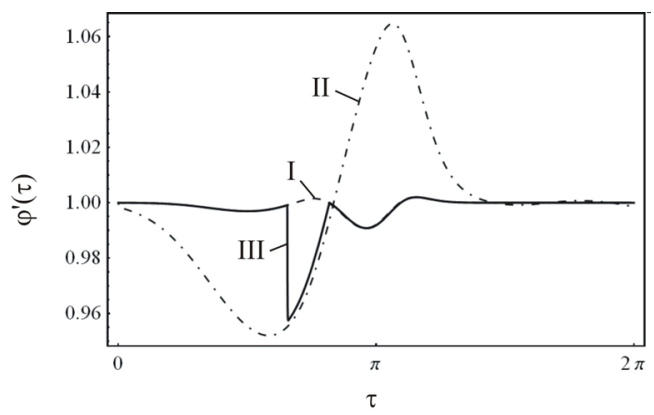

Fig. 5. $\varphi^{\prime}-$ T curves for various values of $\bar{F}(\varphi)$ : I: $\bar{F}(\varphi)=0$, II: $\bar{F}(\varphi)=1$, II: UnitStep function
From the analysis of the curves in Fig. 5 it can be concluded:

- For the case when the cutting force is zero, $\bar{F}(\varphi)=0$, and the motion of the mechanism is without loading, the angular velocity of the leading crank $\mathrm{O}_{1} \mathrm{~A}$ varies as it is shown in Fig. 5 (curve I). Variation of the angular velocity is periodical.

- If it is assumed that the mechanism is loaded with a force $\bar{F}(\varphi)=1$ for all positions of the leading crank, the influence of the force on the angular velocity of the motor motion is extremely high (see curve II in Fig. 5).

- For the case when the cutting process is discontinual and the cutting force has the form Eq. (22) there is a jump in the angular velocity curve (see curve III, Fig. 5).

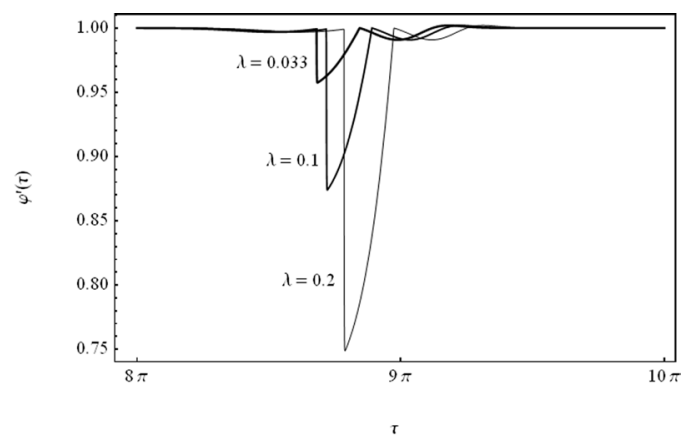

Fig. 6. $\varphi^{\prime}-\tau$ curves for various values of $\lambda$

For this case the influence of the cutting parameter $\lambda$ on the $\varphi^{\prime}-\tau$ is evident (see Fig. 6). The higher the cutting force the higher the velocity variation.

\subsection{Analytical}

Let us consider the case when $M_{0}$ is significantly larger than the parameters $I, \mu, Q$ and $\lambda$ which for the small parameter $\varepsilon<<1$ have the form:

$$
I=\varepsilon I_{1}, \quad \mu=\varepsilon \mu_{1}, \quad Q=\varepsilon Q_{1}, \quad \lambda=\varepsilon \lambda_{1},
$$

Substituting Eq. (32) into Eq. (31) we have:

$$
\begin{aligned}
\left(1-\varphi^{\prime}\right)= & \varepsilon\left(I_{1}+\mu_{1} f^{2}(\varphi)\right) \varphi^{\prime \prime}+\varepsilon \mu_{1} f(\varphi) \frac{d f(\varphi)}{d \varphi} \varphi^{\prime 2}+ \\
& +\varepsilon Q_{1} f^{2}(\varphi) \varphi^{\prime}-\varepsilon f(\varphi) \lambda_{1} \bar{F}(\varphi) .
\end{aligned}
$$

Using the series expansion of the variable $\varphi$ and its time derivatives up to the first order of the small parameter, we obtain:

$$
\begin{gathered}
\varphi=\varphi_{0}+\varepsilon \varphi_{1}+\ldots, \\
\varphi^{\prime}=\varphi_{0}{ }^{\prime}+\varepsilon \varphi_{1}{ }^{\prime}+\ldots,
\end{gathered}
$$




$$
\begin{gathered}
\varphi^{\prime \prime}=\varphi_{0}{ }^{\prime \prime}+\varepsilon \varphi_{1}^{\prime \prime}+\ldots, \\
f(\varphi)=f\left(\varphi_{0}+\varepsilon \varphi_{1}\right) \approx f\left(\varphi_{0}\right)+\varepsilon f^{\prime}\left(\varphi_{0}\right) \varphi_{1}, \\
d f(\varphi) / d \varphi=d f\left(\varphi_{0}+\varepsilon \varphi_{1}\right) / d \varphi \approx \\
\approx\left(\frac{d f(\varphi)}{d \varphi}\right)_{\varphi_{0}}+\varepsilon \varphi_{1}\left(\frac{d^{2} f(\varphi)}{d \phi^{2}}\right)_{\varphi_{0}}, \\
\bar{F}(\varphi) \approx \bar{F}\left(\varphi_{0}\right) .
\end{gathered}
$$

Substituting Eq. (34) into Eq. (33) and separating the terms with the same order of small parameter $\varepsilon$ up to the small value of second order, the system of equations follows:

$$
\begin{gathered}
\varepsilon^{0}: \quad 0=1-\varphi_{0}{ }^{\prime}, \\
\varepsilon^{1}: \varphi_{1}{ }^{\prime}=f\left(\varphi_{0}\right) \lambda_{1} \bar{F}\left(\varphi_{0}\right)-\left(I_{1}+\mu_{1} f^{2}\left(\varphi_{0}\right)\right) \varphi_{0}{ }^{\prime \prime}- \\
-\mu_{1} f\left(\varphi_{0}\right)\left(\frac{d f}{d \varphi}\right)_{\varphi_{0}} \varphi_{0}{ }^{\prime 2}-Q_{1} f^{2}\left(\varphi_{0}\right) \varphi_{0}{ }^{\prime} .
\end{gathered}
$$

Solution of Eq. (35) is $\varphi_{0}{ }^{\prime}=1=$ const. which after integration gives:

$$
\varphi_{0}=\tau \text {. }
$$

Substituting Eq. (37) into Eq. (36) we obtain:

$$
\begin{array}{rl}
\varphi_{1}{ }^{\prime}=-\mu_{1} & f\left(\varphi_{0}\right)\left(\frac{d f}{d \phi}\right)_{\phi_{0}}-Q_{1} f^{2}\left(\varphi_{0}\right)+ \\
& +f\left(\varphi_{0}\right) \lambda_{1} \bar{F}\left(\varphi_{0}\right) .
\end{array}
$$

According to Eqs. (37), (38) and (34) the first order approximate analytical solution is:

$$
\varphi^{\prime}(\tau)=1+\left(-\mu f(\tau)\left(\frac{d f}{d \varphi}\right)_{\tau}-Q f^{2}(\tau)+f(\tau) \lambda \bar{F}(\tau)\right) .
$$

The influence of mass and damping parameters, and also of the cutting force on the angular velocity of the leading element is obtained. In Fig. 7 the analytical result Eq. (39) is compared with a numerical one which is valid for differential equation (33). The difference between the results is negligible.

\section{RESULTS}

Let us analyze Eq. (33) and the analytically obtained solution (39). It follows:
- For the mechanism with omitted mass of the leading crank and of the cutting tool, the angular velocity variation is $\varphi^{\prime}=(1+\lambda f \bar{F}(\varphi)) /\left(1+Q f^{2}\right)$. For higher values of coefficient of damping the angular velocity is smaller. The influence of the cutting force $\lambda$ on the angular velocity $\varphi^{\prime}$ is significant: the higher the cutting force, the larger the angular velocity variation.

- If the mass of the cutting tool and the damping coefficient during cutting are omitted, the differential equation depends on the moment of inertia $I$ of the leading crankshaft and on the cutting force $\lambda$ and is $I \varphi^{\prime \prime}=\left(1-\varphi^{\prime}\right)+\lambda f \bar{F}(\varphi)$.

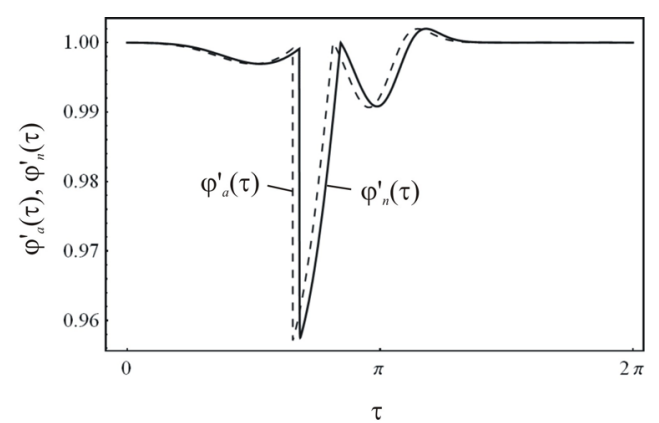

Fig. 7. Comparison of the analytical and numerical $\varphi^{\prime}-\tau$ functions

- For the case when damping is neglected and the cutting force is zero, for the initial angular velocity $\varphi_{0}{ }^{\prime}$ the angular velocity of the leading element varies as $\varphi^{\prime}=1+\left(\varphi_{0}{ }^{\prime}-1\right) \exp (-\tau / I)$. For the steady state motion when time $\tau$ tends to infinity, the angular velocity of the leading element tends to a constant value: $\varphi^{\prime}=1=$ const.

- If the dimensionless driving torque $M_{0}$ is significant in comparison to other parameters of the mechanism, the angular velocity in the first approximation is obtained as $\varphi^{\prime} \approx 1+\varepsilon \varphi_{1}{ }^{\prime}$, where $\varphi_{1}{ }^{\prime}=-\mu f(d f / d \varphi)-Q f^{2}+\lambda f \bar{F}$. For certain parameter values the analytically obtained result is compared with exact numerical one (see Fig. 7). The difference between the results is negligible.

\section{CONCLUSIONS}

The following is concluded:

- The damping during cutting has a significant influence on the angular velocity of the leading element of the cutting mechanism. If the mass of the leading crank and of the cutting tool is quite small it is obvious that for higher values of the 
damping, the angular velocity of the leading crank is smaller.

- The influence of the cutting force on the angular velocity is also significant: the higher the cutting force, the larger is the angular velocity variation.

- The angular velocity variation affects the stability of motion and also the quality of the cutting process. Namely, for high values of angular velocity variation of the leading element, the motor can get from the steady state stable motion into an unstable one. In addition, the higher the cutting force, the cutting process is retarded due to the fact that the averaged velocity is smaller.

\section{ACKNOWLEDGEMENT}

The investigation has been supported by the Ministry of Science of Serbia (Proj. No. ON174028 and No. IT41007) and Province Secretariat for Science and Technological Development, Autonomous Province of Vojvodina (Proj. No 114-451-2094/2011).

\section{REFERENCES}

[1] Artobolevskij, I.I. (1971). Mechanisms in Contemporary Technique. Nauka, Moscow.

[2] Cveticanin, L., Maretic, R. (2000). Dynamic analysis of a cutting mechanism. Mechanism and Machine Theory, vol. 35, p. 1391-1411, DOI:10.1016/S0094114X(00)00007-0.

[3] Metallidis, P., Natsiavas, S. (2003). Linear and nonlinear dynamics of reciprocating engines. International Journal of Non-Linear Mechanics, vol. 38, p. 723-738, DOI:10.1016/S0020-7462(01)00129-9.

[4] Koser, K. (2004). A slider crank mechanism based robot arm performance and dynamic analysis. Mechanism and Machine Theory, vol. 39, p. 169182, DOI:10.1016/S0094-114X(03)00112-5.

[5] Ha, J.L., Fung, R.F., Chen, K.Y., Hsien, S.C. (2006). Dynamic modeling and identification of a slider-crank mechanism. Journal of Sound and Vibration, vol. 289, p. 1019-1044, DOI:10.1016/j. jsv.2005.03.011.

[6] Erkaya, S., Su, S., Uzmay, I. (2007). Dynamic analysis of a slider-crank mechanism with eccentric connector and planetary gears. Mechanism and Machine Theory, vol. 42, p. 393-408, DOI:10.1016/j.mechmachtheory.2006.04.011.

[7] Ogura, M., Daidoji, S. (1982). Application and practice of the double slider crank mechanism for the air compressor. JSME (Japan Society of Mechanical Engineers) International Journal, Series B: Fluids and Thermal Engineering, vol. 48, p. 1483-1491.
[8] Wang, M., Song, Q., Zhong, K. (2012). The double role piston pump based on the symmetrical tears and crank-link-slider mechanism driven by servo motor. Applied Mechanics and Materials, vol. 121-126, p. 2308-2312.

[9] Komatsubara, H., Mitome, K.-I., Sasaki, Y. (2007). A new cutting machine for elliptical cylinder. JSME (Japan Society of Mechanical Engineers) International Journal, Series C: Mechanical Systems, Machine Elements and Manufacturing, vol. 73, p. 891-896.

[10]Kazimierski, Z., Wojewoda, J. (2011). Double internal combustion piston engine. Applied Energy, vol. 88, p. 1983-1985, DOI:10.1016/j. apenergy.2010.10.042.

[11] Amemiya, T., Kawabuchi, I., Ando, H., Maeda, T. (2007) Double-layer slider-crank mechanism to generate pulling and pushing ground. IEEE/RSJ International Conference on Intelligent Robots and Systems, art. no. 4399211, p. 2101-2106.

[12]Amemiya, T., Maeda, T. (2009). Directional force sensation by asymmetric oscillation from a double-layer slider-crank mechanism. Journal of Computing and Information Science in Engineering, vol. 9, art. no. 011001, p. 1-8.

[13] Masia, L., Krebs, H.I., Cappa, P., Hogen, N. (2007). Design and characterization of hand module for whole-arm rehabilitation following stroke. IEEE/ ASME Trans. Mechatronics, vol. 12, p. 399-407.

[14]Xu, F., Wang, X. (2008). Design and experiments on a new wheel-based cale climbing robot. IEEE/ ASME International Conference on Advanced Intelligent Mechatronics, art. no. 4601697, p. 418-423.

[15]Kim, H.S., Park, J.J., Song, J.B. (2008). Safe joint mechanism using double slider mechanism and spring force. $8^{\text {th }}$ IEEE-RAS International Conference on Humanoid Robots 2008, Humanoids 2008, art. no. 4755934, p. 73-78.

[16]Xu, F., Wang, X., Wang, L. (2011). Cable inspection robot for cable-stayed bridges: Design, analysis, and application. Journal of Field Robotics, vol.28, p. 441-459, DOI:10.1002/ rob. 20390.

[17]Ren, T., He, B., Chen, J., Jin, X. (2009). Nonsinusoidal waveform and parameters of distance changeable double-slider crank mechanism for mold. Chinese Journal of Mechanical Engineering, vol. 45, p. 269-273, DOI:10.3901/ JME.2009.12.269.

[18]Sandier, B.Z. (1999). Designing the Mecha-nisms for Automated Machinery. Academic Press, New York. 
[19]Nayfeh, A.H., Mook, D.T. (1979). Nonlinear Oscillations. John Wiley \& Sons, New York.

[20]Zukovic, M., Cveticanin, L. (2007). Chaotic responses in a stable Duffing system of non-ideal type. Journal of Vibration and Control, vol. 13, p. 751-767.

[21]Zukovic, M., Cveticanin, L. (2009). Chaos in nonideal mechanical system with clearance. Journal of Vibration and Control, vol. 15, p. 1229-1246, DOI:10.1177/1077546307072542.

Appendix. DERIVATIVES OF THE KINETIC ENERGY FUNCTION

The derivatives of kinetic energy function Eq. (20) suitable for Lagrange equation (18) are:

$$
\begin{gathered}
\frac{\partial T}{\partial \varphi}=a^{2} m f \frac{d f}{d \varphi} \dot{\varphi}^{2}, \\
\frac{\partial T}{\partial \dot{\varphi}}=J \dot{\varphi}+a^{2} f^{2} \dot{\varphi}, \\
\frac{d}{d t} \frac{\partial T}{\partial \dot{\varphi}}=J \ddot{\varphi}+a^{2}\left(2 f \dot{f} \dot{\varphi}+f^{2} \ddot{\varphi}\right),
\end{gathered}
$$

where ()$=d / d t$. The time derivative of the function $f$ expressed with Eq. (17) is:

$$
\dot{f}=\frac{d f}{d t}=\frac{d f}{d \varphi} \dot{\varphi}
$$

As $f$ explicitely and implicitely depends on the angle $\varphi$ the total derivative of $f$ is:

$$
\frac{d f}{d \varphi}=\frac{\partial f}{\partial \varphi}+\frac{\partial f}{\partial y_{B}} \frac{d y_{B}}{d \varphi}+\left(\frac{\partial f}{\partial y_{E}} \frac{d y_{E}}{d \gamma}+\frac{\partial f}{\partial \gamma}\right) \frac{d \gamma}{d \varphi}
$$

Introducing the notation:

$$
\begin{gathered}
s_{1}=y_{E} \sin \gamma, s_{2}=y_{E}-g \cos \gamma, \\
s_{3}=y_{B} \cos \varphi+l \sin \varphi, s_{4}=y_{B}+a \sin \varphi, \\
s_{5}=p-y_{B}+r \cos \gamma, \\
s_{6}=w \cos \gamma-\left(p-y_{B}\right) \sin \gamma,
\end{gathered}
$$

and substituting into Eq. (17), the function $f$ is:

$$
f(\varphi)=\frac{g}{r} \frac{s_{1}}{s_{2}} \frac{s_{3}}{s_{4}} \frac{s_{5}}{s_{6}} .
$$

The corresponding derivatives of Eq. (A.7) according to Eq. (A.5) are

$$
\begin{gathered}
\frac{\partial f}{\partial \varphi}=\frac{g}{r} \frac{s_{1}}{s_{2}} \frac{s_{5}}{s_{6}} \frac{\partial}{\partial \varphi}\left(\frac{s_{3}}{s_{4}}\right), \\
\frac{\partial f}{\partial y_{E}}=\frac{g}{r} \frac{s_{2}}{\sin \gamma-s_{1}} \frac{s_{3} s_{5}}{s_{4} s_{6}}, \\
\frac{\partial f}{\partial \gamma}=\frac{g}{r} \frac{s_{3}}{s_{4}} \frac{\partial}{\partial \gamma}\left(\frac{s_{1}}{s_{2}} \frac{s_{5}}{s_{6}}\right), \\
\frac{\partial f}{\partial s_{3}}=\frac{g}{r} \frac{s_{1}}{s_{2}} \frac{\partial}{\partial y_{B}}\left(\frac{s_{3}}{s_{4}} \frac{s_{5}}{s_{6}}\right), \\
\frac{\partial s_{1}}{\partial \gamma}=y_{E} \sin \varphi+l \cos \gamma, \quad \frac{\partial s_{2}}{\partial \gamma}=g \sin \gamma, \\
\frac{\partial s_{5}}{\partial \gamma}=-r \sin \gamma, \quad \frac{\partial s_{4}}{\partial \varphi}=a \cos \varphi \\
\frac{\partial s_{6}}{\partial \gamma}=-\left(p-y_{B}\right) \cos \gamma-w \sin \gamma, \\
\frac{\partial s_{3}}{\partial y_{B}}=\cos \varphi, \quad \frac{\partial s_{4}}{\partial y_{B}}=-\frac{\partial s_{5}}{\partial y_{B}}=1 .
\end{gathered}
$$

For Eqs. (3), (6) and (10) the derivatives in angle $\varphi$ are:

$\frac{\partial y_{B}}{\partial \varphi}=-a \frac{s_{5}}{s_{6}}, \quad \frac{\partial y_{B}}{\partial \gamma}=-g \frac{s_{1}}{s_{2}}, \quad \frac{\partial \gamma}{\partial \varphi}=\frac{a}{r} \frac{s_{3}}{s_{4}} \frac{s_{5}}{s_{6}}$.

Substituting Eqs. (A.8) to (A.11) and the also Eqs. (3), (6) and (10) into Eq. (A.5) the $(d f / d \varphi)$ relation is calculated. 